A shorter version of this report will appear in the Proc. of the 50th IEEE Conference on

Decision and Control and European Control Conference, Orlando, FL, December 2012

\title{
Dynamical System Decomposition Using Dissipation Inequalities
}

\author{
James Anderson $^{1 *}$ André Teixeira ${ }^{2}$ Henrik Sandberg ${ }^{2}$ Antonis Papachristodoulou ${ }^{1}$ \\ ${ }^{1}$ Control Group, Department of Engineering Science, University of Oxford, U.K. \\ 2 Automatic Control Laboratory, School of Electrical Engineering, KTH- Royal Institute of Technology Sweden \\ ${ }^{*}$ Corresponding Author E-mail: james. anderson@eng.ox.ac.uk
}

September 6, 2011

\begin{abstract}
In this paper we investigate stability and interaction measures for interconnected systems that have been produced by decomposing a large-scale linear system into a set of lower order subsystems connected in feedback. We begin by analyzing the requirements for asymptotic stability through generalized dissipation inequalities and storage functions. Using this insight we then describe various metrics based on a system's energy dissipation to determine how strongly the subsystems interact with each other. From these metrics a decomposition algorithm is described. Throughout we illustrate these ideas using an RC circuit and a power system example.
\end{abstract}

\section{INTRODUCTION}

The scalable analysis and design of complex dynamical systems is a challenging area in systems and control theory. In this work we describe a set of algorithms that can be used to analyze the stability and characterize the interconnection strength of Linear Time Invariant (LTI) dynamical systems. The methods proposed are based on the notion of dynamical system decomposition [1] and dissipation inequalities with quadratic supply rates [2].

It is frequently the case that many of these systems have an underlying network structure. If the network structure or connection topology is known a priori then this information can be used to help design scalable algorithms for interrogating the system of interest. When the network structure is not known it is important to impose an interconnection topology (decomposition) in order to facilitate further analysis.

In this paper two issues are addressed. We begin by deriving stability criteria for interconnected LTI subsystems using dissipation inequalities and quadratic supply rate functions [2,3]. Conditions for asymptotic stability based on composite Lyapunov functions [4] for passive, bounded gain and input strongly passive subsystems are presented. The subsystems of interest may have been obtained through a decomposition algorithm or they may have a physical realization. In the sequel a method for decomposing networks using the supply rate as a metric for interconnection strength is described and illustrated on an RC network.

System decomposition was first suggested as a framework for handling large-scale systems by Šiljak [5]. However the framework did not provide any insight on how to produce the system decomposition. In recent 
work $[1,6]$ an algorithmic method for producing decompositions based on representing the system as a graph and minimizing the worst case "energy flow" between states was presented. In [7] an alternative approach using Hankel-norm based lumping technique for decomposition was presented.

Using the framework in [1], to decompose a dynamical system model, the system must first be represented as a graph where each node is a state of the system and weighted edges represent state interactions. The decomposition is realized by applying a spectral graph partitioning algorithm $[8,9]$ to the weighted graph. Here we extend this idea to the case where nodes may represent subsystems and edges represent the strength of interaction between subsystems as determined by a given supply rate function. We investigate the physical interpretation of a class of supply rates and how the choice of edge weight affects the system decomposition. We conclude by presenting a clustering based decomposition algorithm.

The paper is organized as follows: In Section 2 we introduce the necessary background material and provide two motivating examples. Section 3 uses quadratic supply rate functions to derive stability criteria using passivity, input strict passivity and finite-gain arguments. In Section 4 various edge weight metrics are described and a decomposition algorithm presented. A numerical example is given in Section 5 and the paper is concluded in Section 6.

\section{PRELIMINARIES}

\subsection{Notation}

$\mathbb{R}^{n}$ denotes the $n$-dimensional Euclidean space, $\mathbb{R}^{n \times m}$ denotes denotes the set of $n \times m$ real matrices. If $M \in \mathbb{R}^{n \times n}$ and $M=M^{\top}$ then $M>0, M \geq 0$ denote that $M$ is positive definite, positive semidefinte respectively. The maximum singular value of $M$ is denoted by $\bar{\sigma}(M)$. Given $k$ matrices $M_{1}, \ldots, M_{k}$, $\operatorname{diag}\left(M_{1}, \ldots, M_{k}\right)$ denotes the concatenated block diagonal matrix.

\subsection{Storage Functions and Quadratic Supply}

In this paper we consider dissipation inequalities containing quadratic nonnegative storage functions, quadratic supply rates, and the notion of $(Q, S, R)$ dissipativity used by Moylan and Hill [3]. Consider the LTI system

$$
\begin{aligned}
\frac{d}{d t} x(t) \triangleq \dot{x}(t) & =A x(t)+B u(t) \\
y(t) & =C x(t),
\end{aligned}
$$

where $x(t) \in \mathbb{R}^{n}, u(t) \in \mathbb{R}^{p}$, and $y(t) \in \mathbb{R}^{m}$ are the state, input and output vectors, respectively. For the sake of simplicity, we will omit the time argument and refer to the former as simply $x$, $u$, and $y$.

System (1) is said to be dissipative with respect to the supply rate $w(u, y)$ if there exists a continuously differentiable storage function $V: \mathbb{R}^{n} \rightarrow \mathbb{R}$ satisfying the following dissipation inequality

$$
\dot{V}(x) \leq w(u, y),
$$

with $V(0)=0$ and $V(x) \geq 0$ for all $x \neq 0$. We are interested in quadratic supply rate functions of the form

$$
w(u, y)=y^{\top} Q y+2 u^{\top} S y+u^{\top} R u,
$$


with $Q=Q^{\top}$ and $R=R^{\top}$, where $u$ and $y$ form valid input-output pairs $(u, y)$ and $Q, S$ and $R$ are of appropriate dimensions. The LTI system (1) is said to be dissipative if the following holds

$$
\begin{aligned}
V(x) & =x^{\top} P x>0 \quad \text { for all } x \neq 0 \\
\dot{V}(x) & =x^{\top}\left(A^{\top} P+P A\right) x+u^{\top} B^{\top} P x+x^{\top} P B u \\
& \leq w(u, y)
\end{aligned}
$$

where $P=P^{\top}$. Dissipativity can be checked by solving the following LMI

$$
\left[\begin{array}{cc}
A^{\top} P+P A-C^{\top} Q C & P B-C^{\top} S \\
B^{\top} P-S^{\top} C & -R
\end{array}\right] \leq 0
$$

for $P>0$. Through various selections of the matrices $(Q, S, R)$ different properties of (1) can be analyzed such as passivity and bounded gain.

\subsection{Stability of Interconnected Dissipative Systems}

In this section we consider the linear interconnection of $N$ subsystems. Each subsystem $\Sigma_{i}$ is described by

$$
\Sigma_{i}\left\{\begin{array}{l}
\dot{x}_{i}=A_{i} x_{i}+B_{i} u_{i} \\
y_{i}=C_{i} x_{i}
\end{array}\right.
$$

and the interconnection between subsystems is given by

$$
u_{i}=-\sum_{j=1}^{N} H_{i j} y_{j} .
$$

Defining $x=\left[x_{1}^{\top} \cdots x_{N}^{\top}\right]^{\top}, u=\left[u_{1}^{\top} \cdots u_{N}^{\top}\right]^{\top}$, and $y=\left[y_{1}^{\top} \cdots y_{N}^{\top}\right]^{\top}$, the interconnection may be written as $u=-H y$ and the global system dynamics are described by

$$
\begin{aligned}
\dot{x} & =A x-B H C x \\
y & =C x,
\end{aligned}
$$

where $A=\operatorname{diag}\left(A_{1}, \cdots, A_{N}\right), B=\operatorname{diag}\left(B_{1}, \cdots, B_{N}\right)$, and $C=\operatorname{diag}\left(C_{1}, \cdots, C_{N}\right)$.

Assumption 1 Each subsystem $\Sigma_{i}$ is dissipative with respect to a given supply rate $\left(Q_{i}, S_{i}, R_{i}\right)$.

Given the previous assumption, for each subsystem there exists a symmetric matrix $P_{i}>0$ such that the dissipation inequality (2) holds with the $\left(Q_{i}, S_{i}, R_{i}\right)$ supply rate. Therefore the inequality

$$
\sum_{i=1}^{N} \dot{V}_{i}\left(x_{i}\right) \leq \sum_{i=1}^{N} w_{i}\left(u_{i}, y_{i}\right)
$$

holds regardless of the interconnection.

Remark 1 Defining $P=\operatorname{diag}\left(P_{1}, \cdots, P_{N}\right)$ and $V(x)=x^{\top} P x$, we have $\dot{V}(x)=x^{\top}\left(A^{\top} P+P A\right) x+$ $u^{\top} B^{\top} P x+x^{\top} P B u=\sum_{i=1}^{N} \dot{V}_{i}\left(x_{i}\right)$. Furthermore, $w(u, y)=\sum_{i=1}^{N} w_{i}\left(u_{i}, y_{i}\right)$ is given by (3) with $Q=$ $\operatorname{diag}\left(Q_{1}, \cdots, Q_{N}\right), S=\operatorname{diag}\left(S_{1}, \cdots, S_{N}\right)$, and $R=\operatorname{diag}\left(R_{1}, \cdots, R_{N}\right)$. 
Considering the interconnection, inequality (8) may be rewritten as

$$
\sum_{i=1}^{N} \dot{V}_{i}\left(x_{i}\right) \leq y^{\top} \hat{Q} y
$$

where $\hat{Q}=Q-S^{\top} H-H^{\top} S+H^{\top} R H$. A sufficient condition for stability of the global interconnected system follows from (9):

Lemma 1 ( [3]) Assume each system $\Sigma_{i}$ is observable. The global interconnected system is asymptotically stable if $\hat{Q}$ is negative definite. Furthermore, $V(x)=\sum_{i=1}^{N} V_{i}\left(x_{i}\right)=x^{\top} P x$ is a Lyapunov function for the global system, with $P=\operatorname{diag}\left(P_{1}, \cdots, P_{N}\right)$.

\subsection{Algebraic Graph Theory}

Consider an undirected weighted graph $\mathcal{G}(\mathcal{V}, \mathcal{E}, \mathcal{Z})$ where $\mathcal{V}=\left\{v_{1}, \ldots, v_{N}\right\}$ is the set of $N$ vertices or nodes, $\mathcal{E}=\left\{e_{1}, \ldots, e_{M}\right\} \subseteq \mathcal{V} \times \mathcal{V}$ is the edge-set and $\mathcal{Z}=\left\{z_{1}, \ldots, z_{M}\right\}$ where $z_{j}>0$ is the weight of edge $j$. Associated with $\mathcal{G}$ is a symmetric weighted adjacency matrix $\mathcal{A}(\mathcal{G}) \in \mathbb{R}^{N \times N}$ where $[\mathcal{A}(\mathcal{G})]_{i j}>0$ if there exists an edge connecting $v_{i}$ to $v_{j}$. The weighted $N \times N$ Laplacian matrix associated with the graph is defined by $\mathcal{L}(\mathcal{G})=\operatorname{diag}(\mathcal{A}(\mathcal{G}) \mathbf{1})-\mathcal{A}(\mathcal{G})$.

The Laplacian matrix has several interesting properties, for undirected graphs $\mathcal{L}(\mathcal{G}) \geq 0$ and the number of zero eigenvalues is equal to the number of connected components in the graph. The incidence matrix, $\mathcal{C}(\mathcal{G}) \in \mathbb{R}^{N \times M}$ of an undirected graph is defined by assigning an arbitrary direction to each $e_{i} \in \mathcal{E}$ and setting

$$
[\mathcal{C}(\mathcal{G})]_{i j}=\left\{\begin{array}{rl}
1 & \text { if } e_{i} \text { enters } v_{j} \\
-1 & \text { if } e_{i} \text { leaves } v_{j} \\
0 & \text { otherwise }
\end{array} .\right.
$$

The weighted Laplacian can then be equivalently defined using the incidence matrix by $\mathcal{L}=\mathcal{C}(\mathcal{G}) W(\mathcal{G}) \mathcal{C}(\mathcal{G})^{\top}$ where $\mathcal{W}(\mathcal{G}) \in \mathbb{R}^{M \times M}$ is a diagonal matrix and $[\mathcal{W}(\mathcal{G})]_{i i}=z_{i}$ with $z_{i} \in \mathcal{Z}$. Given a graph $\mathcal{G}(\mathcal{V}, \mathcal{E}, \mathcal{Z})$, consider a subset of the vertices $\mathcal{V}_{j} \subset \mathcal{V}$; we call the graph $\mathcal{G}_{j}=\mathcal{G} / \mathcal{V}_{j}$ an induced subgraph of $\mathcal{G}$.

Assume that we have a graph $\mathcal{G}(\mathcal{V}, \mathcal{E}, \mathcal{Z})$ which has been partitioned such that $\mathcal{V}=\mathcal{V}_{1} \bigcup \mathcal{V}_{2}$ and $\mathcal{V}_{1} \cap \mathcal{V}_{2}=\emptyset$. If there exists an edge $e_{k}=\left(v_{i}, v_{j}\right) \in \mathcal{E}$ such that $v_{i} \in \mathcal{V}_{1}$ and $v_{i} \in \mathcal{V}_{2}$ (or vice-versa) then $v_{i}$ and $v_{j}$ are called boundary nodes and belong to the set $\mathcal{V}_{b}^{12}$. The set of edges that connect boundary nodes in $\mathcal{V}_{1}, \mathcal{V}_{2}$ is given by $\mathcal{E}_{c}^{12}$.

When it is clear from the context we will omit the graph argument and simply refer to the adjacency, incidence, weighting and Laplacian matrices by $\mathcal{A}, \mathcal{C}, \mathcal{W}$ and $\mathcal{L}$ respectively. For a thorough overview of algebraic graph theory see [10].

\subsection{Illustrative Examples}

Two illustrative examples are now presented: A network of resistors and capacitors and a power system model.

\subsubsection{RC network}

Consider the global system to be a network composed of resistors and capacitors. This network is represented by the undirected weighted graph $\mathcal{G}(\mathcal{V}, \mathcal{E}, \mathcal{Z})$. In this setting, each vertex $v_{i} \in \mathcal{V}$ corresponds to a 


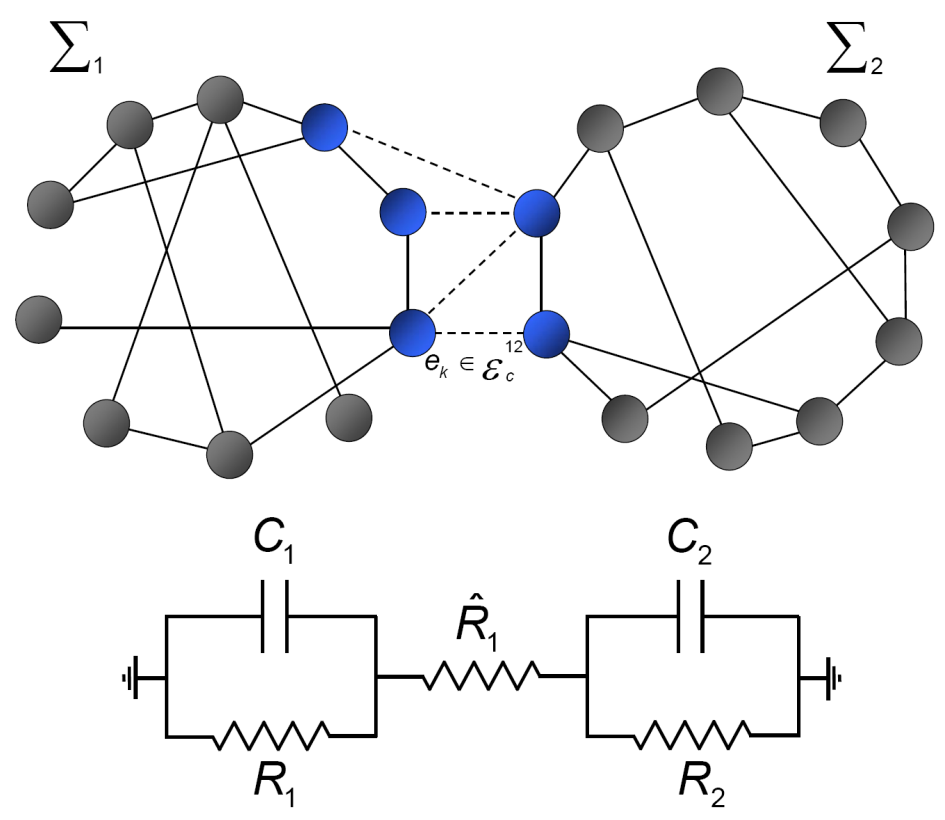

Figure 1: Top: The graph of two interconnected subsystems $\Sigma_{1}, \Sigma_{2}$. Boundary nodes $\mathcal{V}_{b}^{12}$ are coloured blue. Dashed edges, $\mathcal{E}_{c}^{12}$, connect the subsystems via the boundary nodes. Bottom: Two nodes in an RC network, the edge $e_{k} \in \mathcal{E}_{c}^{12}$ corresponds to resistor $\widehat{R}_{1}$ and the vertices correspond to the resistor and capacitors connected in parallel.

capacitor $C_{i}$ in parallel with a resistor $R_{i}$ connected to ground and each edge $e_{k} \in \mathcal{E}$ represents a (possibly negative) resistor $\widehat{R}_{k}$ with $z_{k}=1 / \widehat{R}_{k} \in \mathcal{Z}$ connecting the free terminals of two vertices. This is illustrated in Figure 1. Recalling the model of each component, let all capacitors have unit capacitance and denote $x_{i} \in \mathbb{R}$ as the voltage in capacitor $i$ and $u_{i} \in \mathbb{R}$ the current entering in node $i$. Then each node may be modeled as the first-order system

$$
\dot{x}_{i}=-g_{i} x_{i}+u_{i}, \quad y_{i}=x_{i},
$$

where $g_{i}=1 / R_{i}$. Defining $G=\operatorname{diag}\left(g_{1}, \cdots, g_{N}\right)$ the dynamics of the global network are given by

$$
\dot{x}=-(\mathcal{L}+G) x, \quad y=x .
$$

Consider a subnetwork $\Sigma_{j}$ described by the induced graph $\mathcal{G}_{j}\left(\mathcal{V}_{j}, \mathcal{E}_{j}, \mathcal{Z}_{j}\right)$. The dynamics of this subsystem are described by

$$
\Sigma_{j}\left\{\begin{array}{l}
\dot{x}_{j}=-\left(\mathcal{L}_{j}+G_{j}\right) x_{j}+B_{j} u_{j} \\
y_{j}=B_{j}^{\top} x_{j}
\end{array}\right.
$$

where $u_{j}$ represents the input current to $\Sigma_{j}$ from the rest of the network that enters through the boundary nodes whose dynamics are described by the matrix $B_{j}$. To construct $B_{j}$ : i) Define the following sets $\mathcal{V}_{i}, \mathcal{V}_{j}, \mathcal{E}_{i}, \mathcal{E}_{j}, \mathcal{V}_{b}^{i j}$ and $\mathcal{E}_{c}^{i j}$. ii) From these sets construct an incidence matrix corresponding to $\mathcal{V}_{b}^{i j}, \mathcal{E}_{c}^{i j}$ such that all edges enter $\mathcal{V}_{j}$, iii) $B_{j}$ corresponds to the part of the incidence matrix with nodes belonging to $\mathcal{V}_{j}$.

Defining the storage function for this system as $V_{j}\left(x_{j}\right)=\frac{1}{2} x_{j}^{\top} x_{j}$ we have $\dot{V}_{j}\left(x_{j}\right)=-x_{j}^{\top} \mathcal{L}_{j} x_{j}-$ $x_{j}^{\top} G_{j} x_{j}+u_{j}^{\top} y_{j}$, where the power dissipated on the internal edge resistors, the power dissipated on the node resistors, and the input power to $\Sigma_{j}$ correspond to $-x_{j}^{\top} \mathcal{L}_{j} x_{j},-x_{j}^{\top} G_{j} x_{j}$, and $u_{j}^{\top} y_{j}$, respectively. 
Given $\dot{V}_{j}\left(x_{j}\right)$, two interesting supply rate functions for which the system is dissipative can be immediately identified.

Observation 1 If $\Sigma_{j}$ is stable, then $\Sigma_{j}$ is $(0, I, 0)$-dissipative.

Furthermore, consider $x_{j}^{\top} G_{j} x_{j}=x_{j}^{\top} \tilde{G}_{j} x_{j}+y_{j}^{\top} G_{j}^{b} y_{j}$, where the first term is the power dissipated in the internal node resistors, while the second term corresponds to power dissipated on the boundary node resistors.

Observation 2 If $\mathcal{L}_{i}+\tilde{G}_{j}$ is positive semidefinite, then $\Sigma_{j}$ is $\left(-G_{j}^{b}, I, 0\right)$-dissipative.

\subsubsection{Power network}

A simplified model of an interconnection of synchronous power system generators can be represented by a weighted graph where each vertex corresponds to a bus with a rotating mass, the generator or load, and each edge corresponds to a transmission line connecting two different buses. Denote $\theta_{i} \in \mathbb{R}$ as the angular position of mass $i$ and $u_{i} \in \mathbb{R}$ the electrical power entering in bus $i$ and let $m_{i}>0$ and $d_{i}>0$ be the inertia and damping factor of the rotating mass in $i$, respectively. Using the swing-equation model [11], each node may be modeled as the second order system

$$
m_{i} \ddot{\theta}_{i}=-d_{i} \dot{\theta}_{i}+u_{i}, \quad y_{i}=\left[\dot{\theta}_{i} \theta_{i}\right]^{\top}
$$

where $u_{i}=-\sum_{j} \mathcal{A}_{i j} \sin \left(\theta_{i}-\theta_{j}\right)$. Defining $M=\operatorname{diag}\left(m_{1}, \cdots, m_{N}\right), D=\operatorname{diag}\left(d_{1}, \cdots, d_{N}\right)$, and assuming $M=I$, the linearized dynamics of the global network of subsystems of the form (13) can be written as

$$
\ddot{\theta}=-D \dot{\theta}-\mathcal{L} \theta, \quad y=\left[\dot{\theta}^{\top} \theta^{\top}\right]^{\top} .
$$

Consider a subnetwork of (14) denoted by $\Sigma_{j}$ described by the induced graph $\mathcal{G}_{j}\left(\mathcal{V}_{j}, \mathcal{E}_{j}, \mathcal{Z}_{j}\right)$. The dynamics of this subsystem are described by

$$
\begin{aligned}
\ddot{\theta}_{j} & =-D_{j} \dot{\theta}_{j}-\mathcal{L}_{j} \theta_{j}+B_{j} u_{j} \\
y_{j} & =\left[\begin{array}{cc}
B_{j}^{\top} & 0 \\
0 & B_{j}^{\top}
\end{array}\right]\left[\begin{array}{c}
\dot{\theta}_{j} \\
\theta_{j}
\end{array}\right]
\end{aligned}
$$

where $u_{j}$ represents the input electrical power to $\Sigma_{j}$ from the rest of the network and $B_{j}$ has the same structure as in (12). Defining $x=\left[\dot{\theta}^{\top} \theta^{\top}\right]^{\top}$, the dynamics of $\Sigma_{j}$ may be rewritten in the form of (5). Considering unit masses and defining the storage function for this system as the sum of kinetic and potential energy, $V_{j}\left(x_{j}\right)=V_{k_{j}}+V_{p_{j}}=\frac{1}{2} \dot{\theta}_{j}^{\top} \dot{\theta}_{j}+\frac{1}{2} \theta_{j}^{\top} \mathcal{L}_{j} \theta_{j}$, we have $\dot{V}_{j}\left(x_{j}\right)=-\dot{\theta}_{j}^{\top} D_{j} \dot{\theta}_{j}+u_{j}^{\top} B_{j}^{\top} \dot{\theta}_{j}$, where the power dissipated on the dampers and the input power to $\Sigma_{j}$ correspond to the two terms on the right, respectively.

Observation $3 \Sigma_{j}$ is $\left(0, S_{j}, 0\right)$-dissipative with $S_{j}=\left[\begin{array}{ll}I & 0\end{array}\right]$.

In [12] the dissipative properties of more detailed models of synchronous generators are discussed.

\section{STABILITY ANALYSIS FOR DECOMPOSED SYSTEMS}

The goal of system decomposition is to provide tractable computational analysis tools for analyzing dynamical systems that have a large state dimension. The idea is to take a system model $\dot{x}=A x$ with $x \in \mathbb{R}^{n}$ 
where $n$ is large and decompose it into the form of (5) where composite methods can then be used to infer stability of the original system through less demanding computational analysis of the subsystems.

In this section we describe some stability results based on composite methods that verify the stability of the original system through subsystem Storage functions. We assume that a decomposition algorithm has already been applied, and the subsystems created. In Section 4 we discuss some possible algorithms for decomposition.

Consider the LTI system $\Sigma$ :

$$
\Sigma\left\{\begin{array}{l}
\dot{x}=A x, \quad x(0)=x_{0} \\
y=x
\end{array}\right.
$$

with $x \in \mathbb{R}^{n}$. Now assume that it has been decomposed into two subsystems connected in feedback

$$
\Sigma_{1}\left\{\begin{array}{l}
\dot{x}_{1}=A_{11} x_{1}+u_{1} \\
u_{1}=A_{12} x_{2} \\
y_{1}=x_{1}
\end{array}, \Sigma_{2}\left\{\begin{array}{l}
\dot{x}_{2}=A_{22} x_{2}+u_{2} \\
u_{2}=A_{21} x_{1} \\
y_{2}=x_{2}
\end{array}\right.\right.
$$

where the state vector has been permuted such that $x=\left[x_{1}^{\top}, x_{2}^{\top}\right]^{\top}$ and $x_{1} \in \mathbb{R}^{n_{1}}, x_{2} \in \mathbb{R}^{n_{2}}, n_{1}+n_{2}=n$ and no state belongs to multiple subsystems.

Remark 2 Assume system $\Sigma$ has been decomposed into $\Sigma_{1}$ and $\Sigma_{2}$ which are dissipative w.r.t. the quadratic supply rates $w_{1}\left(u_{1}, y_{1}\right)$ and $w_{2}\left(u_{2}, y_{2}\right)$ of the form (3) respectively. If $w_{1}\left(u_{1}, y_{1}\right)+w_{2}\left(u_{2}, y_{2}\right)<0$ for all input output pairs then the sum of the Storage functions $V_{1}\left(x_{1}\right)+V_{2}\left(x_{2}\right)$ is a Lyapunov function that proves that the equilibrium point of (15) is asymptotically stable. This is a direct application of Lemma 1.

Note that this and all further results generalize to the case where $\Sigma$ can be decomposed into multiple subsystems. For the sake of clarity we focus here on the case of two interacting subsystems.

If we assume a generic interconnection structure for $\Sigma_{1}, \Sigma_{2}$ of the form

$$
\left[\begin{array}{l}
u_{1} \\
u_{2}
\end{array}\right]=\left[\begin{array}{ll}
H_{11} & H_{12} \\
H_{21} & H_{22}
\end{array}\right]\left[\begin{array}{l}
y_{1} \\
y_{2}
\end{array}\right]
$$

then the right hand side of $\dot{V}_{1}\left(x_{1}\right)+\dot{V}_{2}\left(x_{2}\right) \leq w_{1}\left(u_{1}, y_{1}\right)+w_{2}\left(u_{2}, y_{2}\right)$ with $\left(u_{i}, y_{i}\right)$ obtained from the decomposition and interconnection matrix (17) is given by

$$
\left[\begin{array}{c}
x_{1} \\
x_{2}
\end{array}\right]^{\top}\left[\begin{array}{cc}
\left(H_{11}^{\top} R_{1} H_{11}+H_{11}^{\top} S_{1}+S_{1}^{\top} H_{11}+\right. & \left(H_{11}^{\top} R_{1} H_{12}+S_{1}^{\top} H_{12}+\right. \\
\left.+H_{21}^{\top} R_{2} H_{21}+Q_{1}\right) & \left.+H_{21}^{\top} S_{2}+H_{21}^{\top} R_{2} H_{22}\right) \\
\star & \left(H_{22}^{\top} R_{2} H_{22}+H_{22}^{\top} S_{2}+S_{2}^{\top} H_{22}+\right. \\
& \left.+H_{12}^{\top} R_{1} H_{12}+Q_{2}\right)
\end{array}\right]\left[\begin{array}{l}
x_{1} \\
x_{2}
\end{array}\right] .
$$

One interpretation of dynamical system decomposition is to determine the form of the the interconnection matrix $H$ in order to make (18) negative definite.

By appropriate choice of the $Q, S, R$ matrices, the supply functions (3) can represent passivity, finitegain and dissipativity, each of which alters the structure of (18). The remainder of this section examines each of these cases in turn and provides stability tests for (15) based on its decomposed subsystems (16).

\subsection{Passivity}

An LTI system of the form (5) is said to be passive if it is dissipative with respect to supply rate (3) with $Q_{i}=0, S_{i}=I, R_{i}=0$ and LMI (4) is feasible. Assume that $\Sigma$ has been decomposed into $\Sigma_{1}, \Sigma_{2}$ (which is 
equivalent to (5)). Substituting the appropriate matrices into the supply rate functions, we see from Equation (18) that we require

$$
\left[\begin{array}{cc}
H_{11}^{\top} S_{1}+S_{1}^{\top} H_{11} & S_{1}^{\top} H_{12}+H_{21}^{\top} S_{2} \\
\star & H_{22}^{\top} S_{2}+S_{2}^{\top} H_{22}
\end{array}\right]<0,
$$

where from (16) we have that

$$
H=\left[\begin{array}{cc}
0 & A_{12} \\
A_{21} & 0
\end{array}\right]
$$

With this interconnection structure and system decomposition the diagonal block entries in (19) are zero and the off diagonal blocks are given by $A_{12}+A_{21}^{\top}$ and its transposition. In this form (19) cannot be negative definite as its eigenvalues will be real and symmetric about the imaginary axis. This problem can be alleviated if we consider a slight modification to the decomposition described by (16) by imposing a further decomposition on the drift matrices $A_{i i}$ and include a feedback term. The new decomposition for $\Sigma_{1}$ is

$$
\widehat{\Sigma}_{1}\left\{\begin{array}{l}
\dot{x}_{1}=\epsilon_{1} A_{11} x_{1}+u_{1} \\
u_{1}=A_{12} x_{2}+\delta_{1} A_{11} x_{1} \\
y_{1}=x_{1}
\end{array}\right.
$$

where $\epsilon_{1}+\delta_{1}=1$ and we assume all matrices are of compatible dimension. In the same manner $\widehat{\Sigma}_{2}$ can be constructed. The LMI (19) is then replaced by

$$
\left[\begin{array}{cc}
\delta_{1}\left(A_{11}+A_{11}^{\top}\right) & A_{12}+A_{21}^{\top} \\
\star & \delta_{2}\left(A_{22}+A_{22}^{\top}\right)
\end{array}\right]<0
$$

When $\widehat{\Sigma}_{1}, \widehat{\Sigma}_{2}$ are dissipative with respect to $(0, I, 0)$ and LMI (21) is feasible the original system (15) is stable as verified by the Lyapunov function $V(x)=V_{1}\left(x_{1}\right)+V_{2}\left(x_{2}\right)$. An alternative approach is to select $\epsilon_{i}, \delta_{i}$ arbitrarily (ensuring $\epsilon_{1}+\delta_{1}=1$ ) and using the modified decomposition $\widehat{\Sigma}$ solve LMI (19) where the decision variables are the diagonal matrices $S_{i}>0$. Such an approach is possible because any system that is dissipative with respect to $(0, I, 0)$ is also dissipative with respect to any $(0, X, 0)$ supply rate with $X>0$ diagonal.

\subsection{Finite Gain}

For LTI systems the $\mathcal{L}_{2}$ gain from input to output of a system in the form of (5) can be calculated by solving:

$$
\begin{aligned}
\min & \gamma_{i} \\
\text { s.t. } & {\left[\begin{array}{cc}
A_{i}^{\top} P_{i}+P_{i} A_{i}+C_{i}^{\top} C_{i} & P_{i} B_{i} \\
B_{i}^{\top} P_{i} & -\gamma_{i}^{2} I
\end{array}\right] \leq 0 } \\
& P_{i}>0, \quad \gamma_{i}>0 .
\end{aligned}
$$

The $\mathcal{L}_{2} \rightarrow \mathcal{L}_{2}$ gain is then given by $\gamma_{i}$ [13]. For two systems connected in feedback, the small gain theorem [4] states that if $\gamma_{1} \gamma_{2}<1$ then the feedback connection is stable. A generalization of the small gain theorem for networks is given in [14]. Following from LMI (22) it can be seen that the supply rate functions associated with finite gain analysis are given by $\left(-I, 0, \gamma_{i}^{2} I\right)$ for $i=1,2$. 
Substituting the appropriate $Q, S, R$ matrices and interconnection structure (assuming no feedback) into (18) produces the following stability requirement:

$$
\begin{aligned}
& {\left[\begin{array}{cc}
\gamma_{2}^{2} A_{21}^{\top} A_{21} & 0 \\
0 & \gamma_{1}^{2} A_{12}^{\top} A_{12}
\end{array}\right]-\left[\begin{array}{cc}
I & 0 \\
0 & I
\end{array}\right]<0} \\
& \Leftrightarrow \bar{\sigma}\left(\left[\begin{array}{cc}
\gamma_{2} I & 0 \\
0 & \gamma_{1} I
\end{array}\right]\left[\begin{array}{cc}
A_{21} & 0 \\
0 & A_{12}
\end{array}\right]\right)<1
\end{aligned}
$$

The stability condition (23) is stated formally in the following lemma.

Lemma 2 Assume system (15) has been decomposed into the subsystems given in (16). Further assume that the subsystems are dissipative with respect to $S_{i}=0, Q_{i}=-I$ and $R_{i}=\gamma_{i}^{2} I$ where $\gamma_{i}$ denotes the $\mathcal{L}_{2}$-norm of subsystem $i$. Then if $\max \left\{\gamma_{2} \bar{\sigma}\left(A_{21}\right), \gamma_{1} \bar{\sigma}\left(A_{12}\right)\right\}<1$ system (15) is asymptotically stable as verified by the Lyapunov function $V_{1}\left(x_{1}\right)+V_{2}\left(x_{2}\right)$.

Lemma 2 and Equation (23) provide a nominal stability test for the decomposed subsystems. What would be desirable is to determine the maximum $\mathcal{L}_{2}$ gains (i.e. $\gamma_{i}$ 's) such that (23) holds. Such a characterization would provide a robustness measure for the decomposed system. In addition it provides a measure of how good the decomposition is. From the equivalence relation in (23) the maximum achievable $\gamma$ 's denoted by $\hat{\gamma}$ that satisfy the stability requirement in Lemma 2 are given by $\hat{\gamma}_{1}=\bar{\sigma}\left(A_{12}\right)^{-1}$ and $\hat{\gamma}_{2}=\bar{\sigma}\left(A_{21}\right)^{-1}$.

If we consider more generic supply rates with $\left(-\kappa I, 0, \kappa \gamma^{2} I\right), \kappa>0$ instead of $\left(-I, 0, \gamma^{2} I\right)$ then it is possible to strengthen Lemma 2. Observe that if a system is dissipative w.r.t. $\left(-I, 0, \gamma^{2} I\right)$ then it is also dissipative w.r.t. $\left(-\kappa I, 0, \kappa \gamma^{2} I\right)$ for any $\kappa>0$. Taking this into consideration we obtain the following result:

Lemma 3 If there exists a scalar $\kappa>0$ such that $\Sigma_{1}$ and $\Sigma_{2}$ are dissipative w.r.t. $\left(-\kappa I, 0, \kappa \gamma_{1}^{2} I\right)$ and $\left(-\kappa I, 0, \kappa \gamma_{2}^{2} I\right)$ respectively then system (15) is stable if max $\left\{\kappa \gamma_{1} \bar{\sigma}\left(A_{21}\right), \kappa^{-1} \gamma_{2} \bar{\sigma}\left(A_{12}\right)\right\}<1$.

Note that when $\bar{\sigma}\left(A_{21}\right)=\bar{\sigma}\left(A_{12}\right)$, Lemma 3 is equivalent to the small gain condition.

\subsection{Input Strong Passivity}

A system of the form (15) is said to be input strongly passive if $\int_{0}^{T} u^{\top} y-\eta u^{\top} u d t \geq 0$ for all $T \geq 0$, $x(0)=0$ with a dissipation rate $\eta<0$ [13]. The maximum dissipation achievable is the largest $\eta$ such that the integral inequality above holds, this can be computed by solving the LMI

$$
\begin{array}{ll}
\max & \eta \\
\text { s.t. } & {\left[\begin{array}{cc}
A^{\top} P+P A & P B-C^{\top} \\
B^{\top} P-C & 2 \eta I
\end{array}\right]<0} \\
& P>0, \quad \eta<0 .
\end{array}
$$

Setting $\tau=-2 \eta$ we obtain a $Q, S, R$ supply rate of $(0, I,-\tau I)$. Substituting the appropriate supply rate functions $\left(0, I,-\tau_{i} I\right)$ corresponding to $\Sigma_{1}$ and $\Sigma_{2}$ into Equation (18) the requirement for $V_{1}\left(x_{1}\right)+V_{2}\left(x_{2}\right)$ to be a composite Lyapunov function for (15) becomes

$$
\left[\begin{array}{cc}
\tau_{2} A_{21}^{\top} A_{21} & A_{12}+A_{21}^{\top} \\
A_{12}^{\top}+A_{21} & \tau_{1} A_{12}^{\top} A_{12}
\end{array}\right]<0
$$


when $H_{11}=H_{22}=0$. The LMI (25) is never feasible as the elements on the diagonal will always be positive definite. However if we allow for a feedback term in each subsystem by modifying the decomposition according to (20) then it is possible to make (18) negative definite. The modified LMI is easily obtained from (18) but is omitted for lack of space.

Although (25) can never be satisfied it does provide useful insight into the decomposition problem. Factoring (25) into

$$
\begin{array}{r}
{\left[\begin{array}{cc}
A_{21} & 0 \\
0 & A_{12}
\end{array}\right]^{\top}\left[\begin{array}{cc}
\tau_{2} & 0 \\
0 & \tau_{1}
\end{array}\right]\left[\begin{array}{cc}
A_{21} & 0 \\
0 & A_{12}
\end{array}\right]} \\
+\left[\begin{array}{cc}
0 & A_{21}^{\top}+A_{12} \\
A_{12}^{\top}+A_{21} & 0
\end{array}\right]<0
\end{array}
$$

we see that a heuristic that could be incorporated into a decomposition scheme that aims to provide a composite Lyapunov function for (15) with dissipative subsystems is

$$
\min _{A_{21}, A_{12}} \bar{\sigma}\left(\left[\begin{array}{cc}
\sqrt{\tau_{2}} & 0 \\
0 & \sqrt{\tau_{1}}
\end{array}\right]\left[\begin{array}{cc}
A_{21} & 0 \\
0 & A_{12}
\end{array}\right]\right)
$$

where $A_{12}, A_{21}$ are permuted blocks of $A$.The idea of synthesizing a system decomposition will be discussed further in the following sections.

\subsection{Numerical Example}

Consider the RC network (11) with the matrix $-(\mathcal{L}+G) \triangleq A$ :

$$
A=\left[\begin{array}{rrrr}
-3.5750 & 1.6656 & 0.5422 & 0 \\
1.6656 & -5.9846 & 0.4700 & 1.2597 \\
0.5422 & 0.4700 & -3.7523 & 0.1746 \\
0 & 1.2597 & 0.1746 & -2.8511
\end{array}\right]
$$

which has been decomposed into $\widehat{A}=\Pi A \Pi^{-1}$, where $\Pi$ is a permutation matrix with $\Pi_{14}=\Pi_{23}=\Pi_{31}=$ $\Pi_{42}=1$ and all remaining elements set to zero.

Using LMI (22) to compute the $\mathcal{L}_{2} \rightarrow \mathcal{L}_{2}$ gain of the subsystems we obtain $\gamma_{1}=0.4771, \gamma_{2}=$ 0.3558 which satisfies the stability criteria from Lemma 2. Furthermore for this decomposition the system satisfies the stability criteria for input strong passivity with $\delta_{1}=0.6755, \delta_{2}=0.4555$ and optima values of $\eta_{1}=-0.2281, \eta_{2}=-0.1037$. Passivity was obtained with $\delta_{1}=1.689$ and $\delta_{2}=1.1925$. This system decomposition was found by iterating through all permutation matrices $\Pi$ of order $n$. Clearly such an approach is not feasible for large systems. In the following section an algorithmic method for system decomposition is presented.

\section{DECOMPOSITION}

In this section we study the decomposition of interconnected dissipative subsystems. Consider a subsystem $\Sigma_{i}$ described by (5) with a nonnegative storage function $V_{i}\left(x_{i}\right)$ satisfying (4). Such storage is a scalar measure of the subsystem's state, which could be thought of as the amount of "abstract energy" stored by the subsystem in its internal state $x_{i}$. 
Assuming $\Sigma_{i}$ to be dissipative with respect to a given supply rate, the dissipation inequalities (4) indicate that the supply rate upper bounds the rate of change of the storage and thus, indirectly, the change of the subsystem's state. By estimating various forms of supply interchanges between subsystems we can evaluate which subsystems interact most strongly with each other using the supply rate upper bound as an indication of the worst case (strongest) interaction.

\subsection{Directed Supply Measure}

Without loss of generality, suppose the global system is composed of two interconnected subsystems, $\Sigma_{1}$ and $\Sigma_{2}$. These subsystems could be seen as one subsystem $\Sigma_{1}$ and the rest of the interconnected subsystems lumped into $\Sigma_{2}$ provided the lumped systems satisfy Assumption 1 . Since $\Sigma_{1}$ has no external inputs, from its view $w_{1}\left(u_{1}, y_{1}\right)$ can be interpreted as the supply rate performed by $\Sigma_{2}$ and the interconnection $H$ on $\Sigma_{1}$. We argue that $w_{1}\left(u_{1}, y_{1}\right)$ could be seen as a measure for directed interaction from $\Sigma_{2}$ and the interconnection to $\Sigma_{1}$ : When $w_{1}\left(u_{1}, y_{1}\right) \approx 0$ with $u_{1}=-H_{11} y_{1}-H_{12} y_{2}, \Sigma_{2}$ and the interconnection do not interact much with $\Sigma_{1}$ over the set of trajectories of the global interconnected system.

Remark $3 \Sigma_{1}$ could be connected to several subsystems. For the previous discussion to hold, one should constrain the supply rate to be separable along the different edges.

The above remark imposes the following constraint:

Assumption 2 Define $\mathcal{E}_{c} \subset \mathcal{E}$ as the set of edges interconnecting the subsystems. For each subsystem $\Sigma_{i}$ we assume the supply rate is separable along the edges, which implies $w_{i}\left(u_{i}, y_{i}\right)=\sum_{e_{k} \in \mathcal{E}_{c}} w_{e_{k}}^{i}\left(u_{i}, y_{i}\right)$.

Remark 4 Define $\mathcal{E}_{c}^{i j} \subseteq \mathcal{E}_{c}$ as the set of edges connecting $\Sigma_{i}$ and $\Sigma_{j}$. Based Assumption 2, the measure for the directed interaction from $\Sigma_{j}$ and the interconnection to $\Sigma_{i}$ is $\sum_{e_{k} \in \mathcal{E}_{c}^{i j}} w_{e_{k}}^{i}\left(u_{i}, y_{i}\right)$.

Taking the RC-network example, consider the subnetwork $\Sigma_{i}$ and the supply rate defined in Observation 1, $w_{i}\left(u_{i}, y_{i}\right)=u_{i}^{\top} y_{i}$. As mentioned in Section 2.5, $u_{i}$ corresponds to the input current flowing into $\Sigma_{i}$, while $y_{i}$ is the voltage at the boundary nodes. Thus $w_{i}\left(u_{i}, y_{i}\right)$ is the electric power flowing into $\Sigma_{i}$. Hence having $w_{i}\left(u_{i}, y_{i}\right)=u_{i}^{\top} y_{i} \approx 0$ would mean that the rest of the network is not supplying nor consuming electric power from $\Sigma_{i}$ and, as a consequence, the internal electric energy of $\Sigma_{i}$ remains unchanged.

Now considering the supply rate in Observation 2, $w_{i}\left(u_{i}, y_{i}\right)=-y_{i}^{\top} G_{i}^{b} y_{i}+u_{i}^{\top} y_{i}$, this function corresponds to the sum of electric power dissipated on the boundary resistors and the electric power flowing into $\Sigma_{i}$. Therefore, having $w_{i}\left(u_{i}, y_{i}\right) \approx 0$ in this case means that, the electric power supplied to $\Sigma_{i}$ from the rest of the network is being dissipated by the boundary resistors. This implies that the electric energy of the internal nodes remains unchanged.

\subsection{Undirected Supply Measure}

With the interconnection of subsystems $\Sigma_{1}$ and $\Sigma_{2}$ and when Assumption 1 holds the total supply rate given by $w_{1}\left(u_{1}, y_{1}\right)+w_{2}\left(u_{2}, y_{2}\right)=-y^{\top} \hat{Q} y \approx 0$ implies that, over the trajectories of the global system, the interconnections $H$ supply to the subsystems is small. Hence $w_{1}\left(u_{1}, y_{1}\right)+w_{2}\left(u_{2}, y_{2}\right)$ could be seen as a measure of undirected interaction, indicating how relevant the interconnection is to the global system dynamics. Additionally, from Lemma 1 having $w_{1}(\cdot)+w_{2}(\cdot)<0$ implies stability of the global system.

Remark 5 As before, one should constrain the supply rates to be separable along the edges as in Assumption 2. The measure for the undirected interaction between $\Sigma_{i}$ and $\Sigma_{j}$ is then $\sum_{e_{k} \in \mathcal{E}_{c}^{i j}}\left[w_{e_{k}}^{i}\left(u_{i}, y_{i}\right)+w_{e_{k}}^{j}\left(u_{j}, y_{j}\right)\right]$. 
Take the RC-network described previously. The metric discussed in this section corresponds to the electric energy dissipated in the interconnecting resistors for Observation 1 and to the electric energy dissipated on the interconnecting and boundary resistors for Observation 2.

\subsection{Computing Edge Weights for Stability}

We now discuss how the previously described measures of interaction, which are time varying functions of the system state, can be condensed to a representative static value for use in a decomposition algorithm such as the one in [6]. Ideally we would like to find "good" decompositions that satisfy the stability criteria defined in Section 3.

Given the global interconnected subsystem (7), with no assumption of stability. Define for each edge $e_{i}$ the supply rate function

$$
w_{e_{i}}(u, y)=y^{\top} Q_{e_{i}} y+2 u^{\top} S_{e_{i}} y+u^{\top} R_{e_{i}} u=y^{\top} \hat{Q}_{e_{i}} y
$$

where $u$ has been eliminated using the interconnection $u=-H y$ and $\hat{Q}_{e_{i}}$ symmetric, corresponding to either the directed or the undirected interaction measure. For instance, taking the RC-network in Figure 1 and considering the undirected interaction measure for the supply rate from Observation $1, w_{i}\left(u_{i}, y_{i}\right)=u_{i}^{\top} y_{i}$, we have

$$
w_{e_{1}}\left(y_{1}, y_{2}\right)=\left[\begin{array}{l}
y_{1} \\
y_{2}
\end{array}\right]^{\top}\left[\begin{array}{cc}
\frac{1}{\widehat{R}_{1}} & -\frac{1}{\widehat{R}_{1}} \\
-\frac{1}{\widehat{R}_{1}} & \frac{1}{\widehat{R}_{1}}
\end{array}\right]\left[\begin{array}{l}
y_{1} \\
y_{2}
\end{array}\right]=\frac{\left(y_{1}-y_{2}\right)^{2}}{\widehat{R}_{1}},
$$

which corresponds to the electric power dissipated by the resistor $\widehat{R}_{1}$.

As mentioned in Section 3 and 4.2, the undirected interaction measure is also related to stability. In fact given a cut $\mathcal{E}_{c}$ and Assumption 2, if the subsystems $\Sigma_{1}$ and $\Sigma_{2}$ are dissipative with nonnegative storage functions characterized by $P_{1}$ and $P_{2}$ respectively, then we have

$$
x^{\top}\left[(A-B H C)^{\top} P+P(A-B H C)\right] x \leq y^{\top}\left(\sum_{e_{k} \in \mathcal{E}_{c}} \hat{Q}_{e_{k}}\right) y
$$

with $P=\operatorname{diag}\left(P_{1}, P_{2}\right)$, from which it follows that the global system is stable if $y^{\top}\left(\sum_{e_{k} \in \mathcal{E}_{c}} \hat{Q}_{e_{k}}\right) y<0$ (see Lemma 1).

As such a cut is not known a priori, we provide heuristics to compute appropriate edge weights. Let $y_{e_{k}}$ be the output of the two nodes incident to the edge $e_{k}$ and define $\tilde{Q}_{e_{k}}$ such that $y^{\top} \hat{Q}_{e_{k}} y=$ $y_{e_{k}}^{\top} \tilde{Q}_{e_{k}} y_{e_{k}}$. For a suitable permutation yielding $\Pi y=\left[y_{e_{k}}^{\top} y_{\mathcal{E} / e_{k}}^{\top}\right]^{\top}$ we have $\tilde{Q}_{e_{k}}$ as the first diagonal block of $\Pi \hat{Q}_{e_{k}} \Pi^{-1}$. For instance, in the RC-network in Figure 1 we have $\tilde{Q}_{e_{k}}=\hat{Q}_{e_{k}}$. A sufficient condition for $\hat{Q}_{\mathcal{E}_{c}}=\sum_{e_{k} \in \mathcal{E}_{c}} \hat{Q}_{e_{k}}<0$ is to require $\tilde{Q}_{e_{k}}<0 \quad \forall e_{k} \in \mathcal{E}$. Furthermore, note that (27) may be thought as the inclusion of an ellipsoid, $-x^{\top}\left[(A-B H C)^{\top} P+P(A-B H C)\right] x$, by another ellipsoid $-x^{\top} C^{\top}\left(\sum_{e_{k} \in \mathcal{E}_{c}} \hat{Q}_{e_{k}}\right) C x$, where the latter corresponds to a sum of ellipsoids. Since the former ellipsoid is only known after the cut, one would like the latter ellipsoid to be as large as possible, as this would increase the set of matrices $P_{1}$ and $P_{2}$ for which such inclusion holds. Therefore, assuming $\hat{Q}_{\mathcal{E}_{c}}<0$ and denoting $\mathcal{P}_{\mathcal{E}_{c}}=\left\{y:-y^{\top} \hat{Q}_{\mathcal{E}_{c}} y \leq 1\right\}$ as the ellipsoid associated with a given cut $\mathcal{E}_{c}$, a suitable partitioning algorithm would solve

$$
\begin{array}{ll}
\max _{\mathcal{E}_{c}} & \operatorname{vol}\left(\mathcal{P}_{\left.\mathcal{E}_{c}\right)}\right) \\
\text { s.t. } & \mathcal{E}_{c} \text { is a cut, }
\end{array}
$$


where $J\left(\mathcal{E}_{c}\right)=\operatorname{vol}\left(\mathcal{P}_{\mathcal{E}_{c}}\right)$ is the utility of a cut $\mathcal{E}_{c}$. Combining these two features, and denoting $\mathcal{P}_{e_{k}}$ as the ellipsoid defined by $\tilde{Q}_{e_{k}}$, the edge weights $J\left(e_{k}\right)=\operatorname{vol}\left(\mathcal{P}_{e_{k}}^{*}\right)$ may be computed by solving

$$
\begin{aligned}
\max _{Q_{e_{k}}, S_{e_{k}}, R_{e_{k}}} & \operatorname{vol}\left(\mathcal{P}_{e_{k}}\right) \\
\text { s.t. } & \tilde{Q}_{e_{k}}<0, R_{e_{k}}=R_{e_{k}}^{\top}, Q_{e_{k}}=Q_{e_{k}}^{\top}
\end{aligned}
$$

which is not a convex problem in $Q_{e_{k}}, S_{e_{k}}$, and $R_{e_{k}}$. The partitioning algorithm would then choose a set of edges forming a cut $\mathcal{E}_{c}$ such that $\sum_{e_{k} \in \mathcal{E}_{c}} J\left(e_{k}\right)$ is maximized.

The volume of an ellipsoid $\mathcal{P}_{e_{k}}$ is proportional to $\sqrt{\operatorname{det}\left(-\tilde{Q}_{e_{k}}^{-1}\right)}$, hence the previous problem will yield a solution such that $\operatorname{det}\left(-\tilde{Q}_{e_{k}}\right)$ is minimized, which implies $y_{e_{k}}^{\top} \tilde{Q}_{e_{k}} y_{e_{k}} \approx 0$. Therefore, since $\operatorname{det}\left(-\tilde{Q}_{e_{k}}\right)$ is the product of the eigenvalues of $-\tilde{Q}_{e_{k}}$, we can instead consider the convex problem

$$
\begin{aligned}
\min _{Q_{e_{k}}, S_{e_{k}}, R_{e_{k}}} & \lambda_{\max }\left(-\tilde{Q}_{e_{k}}\right) \\
\text { s.t. } & \tilde{Q}_{e_{k}}<0, R_{e_{k}}=R_{e_{k}}^{\top}, Q_{e_{k}}=Q_{e_{k}}^{\top},
\end{aligned}
$$

which is related to maximizing the diameter of $\mathcal{P}_{e_{k}}$, and take $J\left(e_{k}\right)=1 / \lambda_{\max }\left(-\tilde{Q}_{e_{k}}^{*}\right)$. Note that this also relates to finding weakly interacting subsystems based on the undirected measure. Therefore, large values of $J\left(e_{k}\right)$ indicate that this is a good edge to cut in a decomposition algorithm and will also help in verifying stability using the criteria in Section 3.

\subsection{Computing Edge Weights for Weakly Connected Systems}

We now instead know the global system is stable and want to decompose it into subsystems that interact weakly over time, for example to facilitate the design of distributed controllers. Consider the global interconnected subsystem (7), assumed to be stable, and define for each edge $e_{i}$ the supply rate function (26). Since $w_{e_{i}}$ is a quadratic function of the global output $y$ and there are no external inputs, one could initialize the global system from different initial conditions and evaluate $w_{e_{i}}$. However in this case, for each initial condition $x_{0}$ we have $w_{e_{i}}$ as a function of time, hence one would still need to evaluate these functions to compute a static value measuring the interactions over time. Instead the total supply defined as $W_{e_{i}}\left(x_{0}\right) \triangleq \int_{0}^{\infty} w_{e_{i}}(t) d t$ is used where the edge weight is computed by evaluating $W_{e_{i}}\left(x_{0}\right)$ for the relevant initial conditions. The following result allows us to compute $W_{e_{i}}\left(x_{0}\right)$ for a given initial condition:

Proposition 1 Assuming the global system (7) is stable, for a given initial condition $x_{0}$ we have $W_{e_{i}}\left(x_{0}\right)=$ $x_{0}^{\top} T_{e_{i}} x_{0}$, where $T_{e_{i}}$ is the Gramian matrix satisfying the Lyapunov equation $(A-B H C)^{\top} T_{e_{i}}+T_{e_{i}}(A-$ $B H C)+C^{\top} \hat{Q}_{e_{i}} C=0$.

Proof 1 We have $W_{e_{i}}\left(x_{0}\right)=\int_{0}^{\infty} y(t)^{\top} \hat{Q}_{e_{i}} y(t) d t=x_{0}^{\top} \int_{0}^{\infty} e^{\left(\bar{A}^{\top} t\right)} C^{\top} \hat{Q}_{e_{i}} C e^{(\bar{A} t)} d t x_{0}$, where $\bar{A}=A-$ $B H C$. Defining $T=\int_{0}^{\infty} e^{\left(\bar{A}^{\top} t\right)} C^{\top} \hat{Q}_{e_{i}} C e^{(\bar{A} t)} d t$, we see this expression resembles the well-known observability Gramian. The rest of the proof follows the characterization of the observability Gramian found in [15].

Note that for finite time horizons $W_{e_{i}}\left(x_{0}\right)$ can be computed by means of simulation as an alternative to solving the observability Gramian. The aim of the decomposition is to identify subsystems that do not 
interact much with each other. Hence a partitioning algorithm would select a set of edges $\mathcal{E}_{c}$ forming a cut such that $\sum_{e_{i} \in \mathcal{E}_{c}} W_{e_{i}}\left(x_{0}\right)$ is close to zero, thus solving

$$
\begin{aligned}
& \min _{\mathcal{E}_{c}}\left|\sum_{e_{i} \in \mathcal{E}_{c}} W_{e_{i}}\left(x_{0}\right)\right| \\
& \text { s.t. } \mathcal{E}_{c} \text { is a cut set, }
\end{aligned}
$$

where we define $J\left(\mathcal{E}_{c}\right)=\left|\sum_{e_{i} \in \mathcal{E}_{c}} W_{e_{i}}\left(x_{0}\right)\right|$ as the cost of a cut for a given initial condition. We now analyze the evaluation of $W_{e_{i}}\left(x_{0}\right)$ for two different sets of initial conditions.

\subsubsection{Worst-case initial condition}

For a given cut $\mathcal{E}_{c}$, the worst-case initial condition is the one maximizing $\left|\sum_{e_{i} \in \mathcal{E}_{c}} W_{e_{i}}\left(x_{0}\right)\right|$ and the cut cost would be given by $J\left(\mathcal{E}_{c}\right)=\max _{\left\|x_{0}\right\|=1}\left|\sum_{e_{i} \in \mathcal{E}_{c}} W_{e_{i}}\left(x_{0}\right)\right|$. Since we do not know the set $\mathcal{E}_{c}$ a priori, the edge weights are also unknown, which would require a combinatorial approach to solve this partitioning algorithm. A possible relaxation decoupling the edge weights from the cut can be made based on the following inequality $\max _{x_{0}}\left|\sum_{e_{i} \in \mathcal{E}_{c}} W_{e_{i}}\left(x_{0}\right)\right| \leq \sum_{e_{i} \in \mathcal{E}_{c}} \max _{x_{0_{i}}}\left|W_{e_{i}}\left(x_{0_{i}}\right)\right|$. Note that in the right hand side the initial condition $x_{0_{i}}$ is dependent only the edge $e_{i}$. Therefore, by defining the new cost function $\bar{J}\left(\mathcal{E}_{c}\right)=\sum_{e_{i} \in \mathcal{E}_{c}} \max _{x_{0_{i}}}\left|W_{e_{i}}\left(x_{0_{i}}\right)\right|$, we obtain weights that only depend on each particular edge, $\bar{J}\left(e_{i}\right)=$ $\max _{x_{0_{i}}}\left|W_{e_{i}}\left(x_{0_{i}}\right)\right|$, and an upper bound on the edge cost $J\left(\mathcal{E}_{c}\right)$. The weight $\bar{J}\left(e_{i}\right)$ can be computed by solving $\max _{\left\|x_{0}\right\|=1}\left|x_{0}^{\top} T_{e_{i}} x_{0}\right|$ where $T_{e_{i}}$ is given by Proposition 1 .

Remark 6 Since $C^{\top} \hat{Q}_{e_{i}} C$ is symmetric, $T$ is also symmetric and thus we have $\max _{x_{0}}\left|x_{0}^{\top} T x_{0}\right|=\max _{i}\left|\lambda_{i}(T)\right|=$ $\left|\lambda^{*}(T)\right|$, where $\left\{\lambda_{i}(T)\right\}$ are the eigenvalues of $T$. Computing the eigenvalue value decomposition of $T$, we conclude $x_{0}^{*}$ is given by the eigenvector associated with $\lambda^{*}(T)$.

\subsubsection{Gaussian initial condition}

We now consider a stochastic description of the initial condition for the global system. Let $x_{0} \sim \mathcal{N}(\bar{x}, \Omega)$. From Proposition 1 it follows that the total supply $W_{e_{i}}\left(x_{0}\right)=x_{0}^{\top} T_{e_{i}} x_{0}$ is a random variable. Hence the cost of a given cut $\mathcal{E}_{c}$ is $J\left(\mathcal{E}_{c}\right)=\left|\mathbb{E}_{x_{0}}\left[\sum_{e_{i} \in \mathcal{E}_{c}} W_{e_{i}}\left(x_{0}\right)\right]\right|$. Using the triangle inequality we obtain the following upper bound of the cut cost $J\left(\mathcal{E}_{c}\right) \leq \sum_{e_{i} \in \mathcal{E}_{c}}\left|\mathbb{E}_{x_{0}}\left[W_{e_{i}}\left(x_{0}\right)\right]\right|=\tilde{J}\left(\mathcal{E}_{c}\right)$. Hence we assign $\tilde{J}\left(e_{i}\right)=\left|\mathbb{E}_{x_{0}}\left[x_{0}^{\top} T_{e_{i}} x_{0}\right]\right|$ as the weight for $e_{i}$, which may be computed using the following result:

Proposition 2 Given $x_{0} \sim \mathcal{N}(\bar{x}, \Omega)$ we have $\mathbb{E}_{x_{0}}\left[x_{0}^{\top} T_{e_{i}} x_{0}\right]=\bar{x}^{\top} T_{e_{i}} \bar{x}+\operatorname{trace}\left(T_{e_{i}} \Omega\right)$.

Proof 2 Direct application of Lemma 3.3 in [16].

\subsection{Decomposition Methods}

Given the aforementioned methods to compute static edge weights, a system decomposition algorithm based on the directed and undirected interaction measures is described.

The directed interaction measure provides two weights, one for each edge direction. Hence it is suitable for clustering algorithms where a given set of nodes $\mathcal{V}_{0}$ is of interest and we want to find $\mathcal{V}_{i}$ such that $\mathcal{V}=\mathcal{V}_{i} \cup \mathcal{V}_{j}, \mathcal{V}_{0} \subseteq \mathcal{V}_{i}$, and $\Sigma_{i}$ is not affected much by $\Sigma_{j}$. A possible algorithm to accomplish this task proceeds as follows: 
1. Set $\mathcal{V}_{i}=\mathcal{V}_{0}$ and define $\mathcal{E}_{c}$ as the edge set connecting nodes from $\mathcal{V}_{j}$ to $\mathcal{V}_{i}$;

2. Compute the directed weight from $\mathcal{V}_{j}$ to $\mathcal{V}_{i}$ for each edge $e_{k} \in \mathcal{E}_{c}$;

3. Pick the set of nodes from $\mathcal{V}_{j}$ that have the largest directed weight, $\overline{\mathcal{V}}_{j}$, and set $\mathcal{V}_{i}^{+}=\mathcal{V}_{i}+\overline{\mathcal{V}}_{j}$;

4. Set $\mathcal{V}_{i}=\mathcal{V}_{i}^{+}$, define the new cut set $\mathcal{E}_{c}$, and repeat from 2 until the interaction measure is below the tolerance level.

The undirected interaction measure provides a single weight, $W_{e_{k}}$, thus can be readily incorporated into the framework presented in [6] which is based on graph partitioning.

\section{EXAMPLE}

Consider an RC-network described by the graph in Figure 1 with dynamics given by (11). Let each node have unit capacitance and resistance and let $[\mathcal{W}]_{i i}=1 / \hat{R}_{i}=0.1 \forall e_{i} \in \mathcal{E}_{c}^{12}$ and $[\mathcal{W}]_{i i}=1 \forall e_{i} \notin \mathcal{E}_{c}^{12}$. For each node $v_{i}$, consider the supply rate defined in Observation $1, w_{i}\left(u_{i}, y_{i}\right)=u_{i} y_{i}$. Recalling that $u_{i}$ is the input current to node $i$ and $y_{i}=x_{i}$ is the voltage at the corresponding capacitor, from Kirchhoff's Current Law we conclude that the supply rate is separable along the edges connected to $v_{i}$, since $w_{i}\left(u_{i}, y_{i}\right)$ is the sum of the input power from each edge. Hence Assumption 2 holds. Following the steps in Section 4 for the undirected measure and the worst-case initial condition approach we compute the edge weights, which correspond to the electric power dissipated at each edge resistor. For the dashed edges we obtain the weights

$$
\bar{J}\left(e_{i} \in \mathcal{E}_{c}^{12}\right)=[0.0579,0.0625,0.0693,0.0623]^{\top},
$$

while $\min _{i} \bar{J}\left(e_{i} \notin \mathcal{E}_{c}^{12}\right)=0.4016$. Applying a spectral graph decomposition algorithm with these edge weights we obtain $\mathcal{E}_{c}^{12}$ as the cut set, as shown in Figure 1.

Considering instead the undirected measure with Gaussian initial condition $x_{0} \sim \mathcal{N}(0, I)$, we obtain the following weights

$$
\tilde{J}\left(e_{i} \in \mathcal{E}_{c}^{12}\right)=[0.0636,0.0670,0.0727,0.0671]^{\top},
$$

and $\min _{i} \tilde{J}\left(e_{i} \notin \mathcal{E}_{c}^{12}\right)=0.4278$. As before, the cut set obtained after spectral decomposition is $\mathcal{E}_{c}^{12}$.

\section{CONCLUSIONS AND FUTURE WORK}

It has been shown how the supply rates of dissipative dynamical systems can be used as a metric for measuring subsystem interaction strength in a networked system. Furthermore, based upon this metric an algorithm for decomposing a networked system was presented and illustrated on a 20 node RC circuit. We also provided stability criteria for the decomposed system based on passivity and bounded gain.

\section{Acknowledgements}

J. Anderson and A. Papachristodoulou gratefully acknowledge funding from the EPSRC grant EP/I031944/1. A. Teixeira and H. Sandberg are supported by the European Commission through the VIKING project and the Swedish Foundation for Strategic Research. 


\section{References}

[1] J. Anderson and A. Papachristodoulou, "A network decomposition approach for efficient sum-ofsquares programming based analysis," in Proceedings of the American Control Conference, Baltimore, $M D, 2010$.

[2] J. C. Willems, "Dissipative dynamical systems. II. Linear systems with quadratic supply rates," Arch. Rational Mech. Anal., vol. 45, pp. 352-393, 1972.

[3] P. J. Moylan and D. J. Hill, "Stability criteria of large-scale systems," IEEE Transactions on Automatic Control, vol. 23, no. 2, pp. 143-149, 1978.

[4] H. K. Khalil, Nonlinear Systems, 3rd ed. Prentice Hall, Inc., 2001.

[5] D. D. Šiljak, Large-scale dynamic systems: Stability and Structure. North-Holland, New York, 1978.

[6] J. Anderson and A. Papachristodoulou, "Dynamical system decomposition for efficient sparse analysis," in Proceedings of the 49th IEEE Conference on Decision and Control, Atlanta, GA, 2010.

[7] H. Sandberg, "Hankel-norm-based lumping of interconnected linear systems," in Proceedings of MATHMOD 2009, Vienna, Austria, 2009.

[8] G. Karypis and V. Kumar, "A fast and high quality multilevel scheme for partitioning irregular graphs," SIAM J. Sci. Comput., vol. 20, no. 1, pp. 359-392, 1998.

[9] M. X. Goemans and D. P. Williamson, "Improved approximation algorithms for maximum cut and satisfiability problems using semidefinite programming," J. ACM, vol. 42, pp. $1115-1145,1995$.

[10] C. Godsil and G. Royle, Algebraic Graph Theory. Springer-Verlag, Inc., 2000.

[11] J. Machowski, J. W. Bialek, and J. R. Bumby, Power System Dynamics: Stability and Control. John Wiley \& Sons, 2008.

[12] A. Giusto, A. Stankovic, and R. Ortega, "Dissipativity properties of detailed models of synchronous generators," in Proceedings of the 47th IEEE Conference on Decision and Control, 2008, pp. 2475 -2480 .

[13] S. Boyd, L. E. Ghaoui, E. Feron, and V. Balakrishnan, Linear matrix inequalities in system and control theory. Philadelphia, PA, USA: Society for Industrial and Applied Mathematics, 1994.

[14] S. Dashkovskiy, B. Rüffer, and F. R. Wirth, "An ISS small gain theorem for general networks," Mathematics of Control, Signals, and Systems, vol. 19, pp. 93-122, 2007.

[15] M. Green and D. J. N. Limebeer, Linear Robust Control. Prentice-Hall, Inc., 1995.

[16] K. J. Åström, Introduction to Stochastic Control Theory. Dover Publications, 2006. 